\title{
A FRAMEWORK FOR MAPPING MULTIMEDIA TO EDUCATIONAL CONCEPTS
}

\author{
Chigozie Onyekaba ${ }^{1}$, Russell Campion ${ }^{2}$, Anthony Atkins ${ }^{3}$ \\ ${ }^{1}$ chigozie.onyekaba@research.staffs.ac.uk Staffordshire University (UNITED KINGDOM) \\ ${ }^{2}$ r.j.campion@staffs.ac.uk Staffordshire University (UNITED KINGDOM) \\ 3 a.s.atkins@staffs.ac.uk Staffordshire University (UNITED KINGDOM)
}

\begin{abstract}
Multimedia provides exciting opportunities to education as it allows educational material to be delivered in a multitude of ways, fine-tuned to enhance the learning experience. By using multimedia in e-learning systems, educational material can be presented in ways that both simplify and enhance learning. Increasingly, educational material developers are taking advantage of multimedia technology to deliver education but use multimedia in a variety of ways that are more based on intuition and personal experience and this often leads to the development of an ineffective learning material. Although there are not many main media prototypes (animation, images, video, text, audio), when examined at sub-prototype level, we find that there are many media types that can be used to present educational information. Given the number of available media sub-prototypes, the choice and mode of delivery of multimedia presents a problem in designing educational material. What media type or combination of types do we use for a given educational concept? What media combinations will improve learning for a particular educational concept? This paper presents a method for the selection of appropriate media for educational material and demonstrates how the method could be effectively used in the design of educational material, suitable for e-learning applications.
\end{abstract}

Keywords: Multimedia, e-learning, media selection, educational material.

\section{INTRODUCTION}

It is widely accepted that learning is improved when multimedia is used in education ([1], [2], [3]). The invention of computers, the internet and multimedia have brought with it the possibility of developing highly effective and easily-accessible learning environments. The invention of these technologies has brought about an increase in multimedia related research. Researchers have sought ways to utilise multimedia to enhance education and learning experience. As a result, a number of theories about the use of multimedia have emerged. While some of these theories focus on the effects of multimedia on learning and how best to take advantage of multimedia, there is little information about the specific media type (or types) suitable for a given concept, in the development of multimedia learning materials. As a result, educational material developers tend to create learning content intuitively and based on their individual experiences [4]. This can sometimes have adverse effects on learning experience. For example, a well-designed interface that makes improper use of media may end up inhibiting learning rather than enhancing learning. In the next section, we discuss some of the theories that have emerged in the field of multimedia learning.

\section{MULTIMEDIA AND LEARNING}

The use of multimedia for presenting information, especially in education, has greatly increased in recent years. This is because learning is deeper when multimedia is used, compared to traditional methods of learning [3]. Using multimedia applications such as learning management systems, intelligent tutoring systems and virtual learning environments, people can now take courses and learn very effectively from virtually anywhere. Examples of such systems are Blackboard, Moodle, Brightspace, etc. The use of multimedia in education brings with it a lot of benefits, which include personalised learning, self-managed and self-paced learning, etc. [5]. A significant amount of research work has focused on multimedia and its effect on the learning process and as a result, various theories about the use of multimedia have emerged. One such theory suggests that people learn better when on-screen text is replaced with audio narrations. This is referred to as the modality effect ([2], [6], [7]). One reason for this is that higher demand is placed on memory when the learners have to joggle between text and image to create a mental representation of them. The modality effect can also be explained by the dual coding theory [8], which is based on the postulation that humans 
generally process information through two channels (verbal and non-verbal). When both channels are utilised simultaneously, learning is usually improved. Although the modality effect is generally accepted by many researchers, it was found not to apply in every situation [9]. The researchers were able to show that in a non-system paced environment, images with on screen text can be as effective as images with audio narrations. The modality effect therefore becomes less observable when learners are allowed more time to learn. This experiment was repeated [10] and the modality effect was still observed. However, conditions under which the modality effect applies were noted. One such condition is when the learner is not allowed extra time to learn. This corresponds with earlier findings [9].

Multimedia research has produced a number of theories such as the modality effect (replacing words with narrations), coherence principle (exclusion of extraneous media), contiguity principle (placement of descriptive text very close to images) [11], however, not much is known about what specific media type is suitable for a given learning concept. There is evidence that some media types and/or combinations appear to be more effective than others in enhancing learning [12]. Findings from research conducted by [12] confirm that choice of media is crucial in multimedia learning and based on observation, this choice is even more crucial when dealing with more complex learning tasks. This suggests that the choice of media in the development of educational material is key to effective learning. The importance of getting media selection right cannot be overemphasised because improper use of media can have an adverse effect on learning experience.

Some guidelines for the selection of media have been produced in the past. For example, guidelines for mapping some information types to media types (text, sound, graphics and motion) have been proposed [13]. The information types used were: - concrete, abstract, spatial temporal, quantitative and covariant relationship. A method for designing multimedia interfaces, also covering media selection was also proposed [14]. The researchers provided advice on media selection based on a set of communication goals which include generalised tasks such as explain, warn, excite and attract. One problem with these guidelines is that they do not adequately take into account the vast amount of media types, particularly subtypes, that are available. For example, Heller's multimedia taxonomy framework [13] made use of only four media types which are motion, graphics, sound and text. Some of these guidelines also apply to the development of general user interfaces [14] and not particularly suited to educational requirements. Media richness theory [15] has been examined in educational multimedia design and was found to be useful in the design of some educational materials [16], however, classifying media as being high or low in richness does not say much about its suitability for a given educational concept, we would argue. It could also lead to the inadvertent use of irrelevant multimedia and multimedia used in that way, may have adverse effects on learning [17].

For educational purposes, a framework covering the media types and subtypes frequently used is much needed to guide the media selection process in the design of educational material. In this paper, we describe a method covering the selection of media for educational material and we demonstrate how it could be used.

\subsection{What is multimedia?}

There is often confusion surrounding the terms media and multimedia. The term media is sometimes used to refer to instructional or informational delivery methods. Two sources of confusion with the term media have been highlighted [18], one of which was a lack of distinction between media and the methods used to present or deliver media. Kozma [19] described a medium as a resource that possesses information presenting attributes or capabilities. These resources are usually used for instructional purposes or to convey information. Examples of the information presenting attributes are video, text, audio and graphics. Multimedia can therefore be said to be a combination of these attributes (or a seamless integration of them [13]) to present information. In this paper, we refer to these media attributes as media types. This is in line with ISO's definition, which refers to media as "different specific forms of presenting information to the human user" and multimedia as "combinations of static and/or dynamic media which can be interactively controlled and simultaneously presented in an application" [20]. So how many media types are there? There are not a lot of main media types but when considered at subtype level, there are quite a lot, and given the vast amount of multimedia materials available, which incorporate a lot of multimedia components (or media types as we call it), there is a need to identify and classify these media types. The next section presents a media taxonomy for the classification of media types. 


\section{MEDIA TAXONOMY}

There are hundreds of specific media types available and with new media types being developed frequently, it may not be feasible to identify all available media types. This presents the need to sort media types into categories which make them easier to work with. For this purpose, we have developed a media taxonomy. Figure 1 shows the five main media types and their first level subtypes. These media types are usually employed in the development of e-learning materials. We also present a brief definition of each of the media types and subtypes shown in Figure 1.

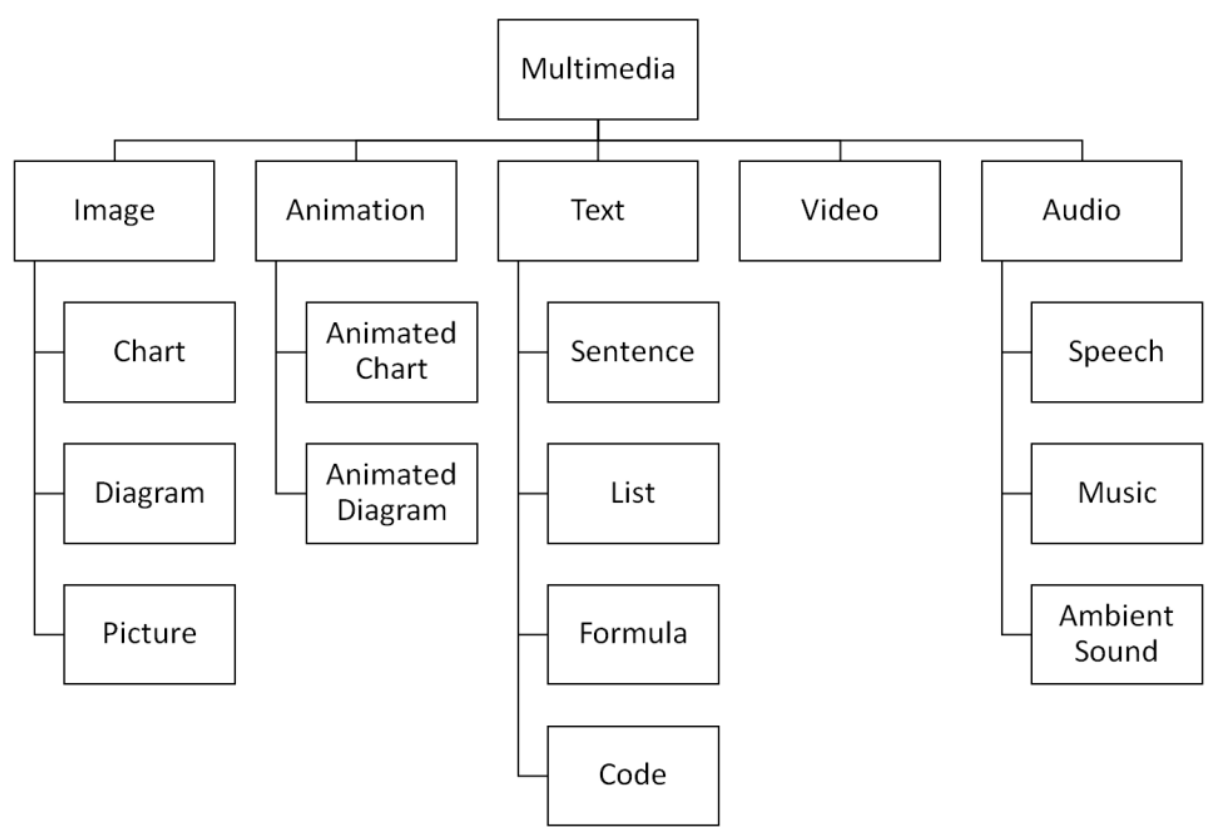

Figure 1: Media Taxonomy Hierarchical Diagram

Image: This media type is visual in nature and is used to record visual perception about something, physical or abstract. Three subtypes of image, as described by this taxonomy, are listed as follows:-

- Chart: A chart is an image type that presents a visual representation of data or anything that is data related. Examples are pie charts, histograms, line charts, scatterplots, etc.

- Diagram: These are images that are used to portray a visual model of something. This something may be physical or abstract. Examples are maps, schematics, etc.

- Picture: A picture is a visual representation of something physical and tangible, usually created by capturing an image with a camera. Examples are photographs, screenshots, etc.

Animation: Animations are, in simple terms, motion images. Images in general are still artefacts but animations usually introduce motion to images, sometimes created by displaying images successively, to create the illusion of motion. This taxonomy presents two subtypes of animation as follows: -

- Animated Chart: This is an animation which takes all the attributes of a chart and adds to it animation. For example, a chart may be animated to illustrate changes in two variables with time. A good example of an animated chart is the motion chart.

- Animated Diagram: This is an animation which has all the attributes of a diagram, with motion added to it. For example, to illustrate the circulation of blood in the human body, a diagram of the heart and blood vessels could be animated. It is important to note that the majority of animations are animated diagrams.

Text: Text is the most widely used media type available. It is, in simple terms, written words. There are forms of use that make it necessary for us to create sub types of text.

- Sentence: This is essentially text that is used to convey information. 
- List: A list is a text type in which items are enumerated. It enhances the presentation of information about items that make up a set or group of something. An example could be a list of guidelines for enhancing software usability.

- Formula: This is a subtype of text which lends use in representing mathematical equations. An example is written as follows: $-V=4 / 3 \pi r^{3}$

- Code: This is a type of text that represents an instruction code such as a computer program. Although it is essentially text, it is usually styled and indented to enhance readability.

Video: A video can be defined as a motion picture. While it may be a little close to an animation, they differ in characteristics. Videos usually contain real objects (but not always). Examples may be a video of a person demonstrating a physical exercise, or the video from a computer screen recording.

Audio: Audio is the media type which presents information that is processed by the auditory channel (or sense of hearing). It is essentially sound, anything we can hear. Let us consider some subtypes of audio, as defined by this taxonomy.

- Speech: Speech is essentially vocal communication or spoken words. We communicate daily using speech. Thoughts, feelings and ideas are usually expressed using this media type and teachers in schools present information, mainly using speech.

- Music: Music is essentially a harmonious audio or sound. Sometimes speech is used to create music but the harmony involved gives it a distinct attribute.

- Ambient Sound: Given the huge variety and variability of audio, we have created this sub type. Ambient sound is essentially everything we hear in our environment. We live in a world full of sound so it is essential that a category is created comprising the sound all around us. Examples are the sound of a dog barking, a vacuum cleaner, a car engine, thunder, etc.

It is expected that every media type (existing and newly created types) will fall into one of these categories. In the next section, we describe a proposed framework for selecting these media types during the development of online educational material.

\section{MEDIA SELECTION}

The selection of appropriate media types for learning involves addressing one of the issues with multimedia design which is matching the media to the message [14]. This ensures effective comprehension of the concepts being learned. The educational material design process usually begins with requirements or task analysis. Task analysis is used to fully establish what the communication goals are, the preconditions, the tasks and subtasks required for the learning process. This can be done by adopting the Task Knowledge Structures approach [21] or Hierarchical Task Analysis [22]. Once the tasks and subtasks have been developed, details about what is intended for the educational material become clearer. The next stage will be to select the appropriate media types to represent the information intended for communication.

\subsection{First level media selection}

In order to guide the process of media selection, we have produced a list of identifiers that map to various suitable media types, outlined in order of appropriateness of the media type. When the task analysis stage is complete, the educational material designer then goes through this list for each task, selecting the set of identifiers that relate to the tasks that emerged from the task analysis stage. These information identifiers are shown in Table 1. A brief explanation of the identifiers is outlined as follows.

Code: This identifier is used for concepts that involve programming. An example is when learning about computer programming and there is a need to show sections of a code for detailed explanation.

Data: Used for concepts about numerical data or where there is a need to present data.

Maths: Used for aspects of learning that involve mathematical statements or expressions. For example, when learning about the mean (average) in statistics, the maths identifier can be used because the mean can be expressed mathematically.

Model: Sometimes, learning concepts cannot be concisely presented without some sort of objectification and/or rescaling. This identifier is used for concepts that can't be presented as is, but 
Table 1: Information identifiers and recommended media types

\begin{tabular}{|l|l|}
\hline Identifier & Recommended Media \\
\hline Code & Source Code \\
\hline Data & Chart, Animated Chart \\
\hline Maths & Formula \\
\hline Model & Diagram, Animated Diagram \\
\hline Motion & Animated Diagram, Video \\
\hline Object & Picture, Diagram, Video, Animated Diagram \\
\hline Reality & Picture, Video, Ambient Sound \\
\hline Requirements & List, Chart \\
\hline Sound & Ambient Sound, Music \\
\hline Message & Sentence, Speech \\
\hline Static & Picture, Diagram \\
\hline Demonstration & Video, Animated Diagram, Animated Chart \\
\hline
\end{tabular}

require a different representation or need to be objectified to highlight important aspects. An example of when this could be used, is when learning about the atom.

Motion: This identifier is used for concepts where the movement of objects is an integral part of the concept being learned. For example, when we learn about planetary rotation, there is motion involved in the concept (the movement of planets) so the motion identifier could be used.

Object: This identifier is used for concepts that are about physical items.

Reality: This identifier is used for concepts about objects which need to be represented as they are. An example is when one needs to learn to identify a physical item.

Requirements: This identifier is used for learning concepts that may need to highlight a set of rules or procedures about something. An example is when discussing ingredients for a recipe.

Sound: This identifier is used for concepts where learning can only occur through the auditory channel. An example may be when learning about the sound of animals, because such cannot easily represented in text.

Message: Used in cases that involve presenting descriptive or informational messages.

Static: This identifier may be used for concepts that do not involve the movement of things or objects. An example of when static may be used is when learning about different breeds of a dog.

Demonstration: This identifier is used when an activity or process needs to be exhibited. For example, we use this identifier when we need to show how something works.

Once the relevant information identifiers have been selected, the educational material designer simply picks out the most occurring media type from the list of recommended media types. If there is a multimodal outcome (by this we mean cases where there are two most occurring media types from the recommendation), the designer would select the most occurring media type that appears first in the recommended media types. As mentioned earlier, the recommended media types have been listed in order of appropriateness but this order is only useful where there is a multimodal media type outcome.

\subsection{Second level media selection}

For some media types, a second level media selection may be required. These media types are: Chart, Diagram, Animated Chart, and Animated Diagram.

We have provided two second level information identifiers, one for charts (and animated charts) and the other for diagrams (and animated diagrams).

The identifiers and recommended media types for charts are provided in Table 2 as follows: - 
Table 2: Information identifiers and recommended media types for diagrams

\begin{tabular}{|l|l|}
\hline Identifiers & Recommended Diagrams \\
\hline process & Flow Chart, Cycle Diagram \\
\hline cycle & Cycle Diagram \\
\hline algorithm & Flow Chart \\
\hline chronology & Timeline Chart \\
\hline hierarchy & Hierarchical Diagram, Organisational Chart \\
\hline relationship & Hierarchical Diagram, Concept Map, Mindmap, Euler Diagram, Venn Diagram \\
\hline concept & Concept Map, Mindmap \\
\hline object & Schematic Diagram, UML Diagram \\
\hline component & Schematic Diagram, UML Diagram \\
\hline system & Schematic Diagram, UML Diagram \\
\hline set & Euler Diagram, Venn Diagram \\
\hline software & UML Diagram \\
\hline data & Heatmap, Thematic Map \\
\hline spatial & Physical Map, Thematic Map, Heatmap, Schematic Diagram \\
\hline location & Physical Map, Thematic Map \\
\hline
\end{tabular}

Process: This identifier represents concepts that involve the description of activities or tasks that occur in predefined steps or sequence.

Cycle: This identifier represents concepts that describe activities or tasks that occurs cyclically.

Algorithm: This identifier represents concepts that involve the description of steps required to complete a process.

Chronology: This identifier represents concepts that describe events and their time of occurrence.

Hierarchy: This information identifier represents concepts that describe a hierarchical system of operation or organisation.

Relationship: This information identifier represents concepts that need to highlight links between entities or the concepts being described.

Concept: This information identifier can be used when one needs to describe or structure ideas.

Object: This information identifier can be used when details about an object (whether physical or abstract) needs to be presented.

Component: This identifier is used when there is need to show the constituents of a system.

System: This information identifier can be used when an organised method of operation needs to be described. This could be a physical (tangible) system or an abstract system.

Set: This identifier can be used when there is need to show group relationships for items or things.

Software: This identifier can be used when information about software needs to be described.

Data: Most diagrams do not depict data but a few could show data in addition to the information model they display. This information identifier can be used when data needs to be depicted in a diagram.

Spatial: This information identifier can be used for concepts that have spatial attributes like position, area and size.

Location: This information identifier can be used when locational information needs to be presented.

The identifiers and recommended media types for charts are provided in the Table 3 as follows: - 
Table 3: Information identifiers and recommended media types for charts

\begin{tabular}{|l|l|}
\hline Identifiers & Recommended Charts \\
\hline trend & Time Series Chart, Line Chart, Area Chart \\
\hline record & Table \\
\hline properties & Scatterplot, Table \\
\hline comparison & Area Chart \\
\hline correlation & Scatterplot \\
\hline relationship & Scatterplot, Table \\
\hline discrete & Bar Chart, Pie Chart, Dot Plot \\
\hline continuous & Histogram \\
\hline
\end{tabular}

Trend: This identifier is used when it is necessary to depict the change of data over time.

Record: This identifier is used when a record or records of something needs to be shown.

Properties: This identifier is used to present information about entities that possess attributes.

Comparison: This identifier is used when two or more data points need to be compared.

Correlation: This identifier is used when there's need to visualise interrelationship between factors.

Relationship: This identifier may be used when connection between data needs to be examined.

Discrete: This identifier is used for concepts that deal with distinct (or categorical) data.

Continuous: This identifier is used for concepts that deal with data that has a range.

Where a second level media selection is required, the educational material designer would go through the list of information identifiers for charts or for diagrams and select the relevant media type using the same rules of selection as described in the selection of the main media types. We have modelled the media selection process for this framework, in a flow chart in Figure 2.

In this section, we have presented a number of identifiers and a description them, including the recommended media types for these identifiers. In the next section, we present a sample multimedia material development to show how this framework can be used.

\section{MULTIMEDIA MATERIAL DEVELOPMENT}

In order to demonstrate how this framework can be used, we would walk through the process of developing a simple educational material about planetary revolution.

\subsection{Task analysis}

The first stage is to do a task analysis (see [21] or [22] for more information). For the purpose of this demonstration, we would keep the tasks as simple as possible. The following are the tasks for our educational material.

1. Define planetary revolution: For this task, we want to present a definition and an explanation of what planetary revolution is.

2. Demonstrate how planetary revolution works: For this task we want to show in detail how planetary revolution work.

\subsection{Selecting the identifiers and media}

To select the identifiers, we first examine the learning tasks.

Task 1 (Define planetary revolution): This task requires a definition of planetary revolution. From our list of information identifiers, we would select "message". This is because a definition involves presenting informational messages. From the table, we have "sentence" and "speech" as the recommended media types. In this case, we would select sentence because the recommended media 


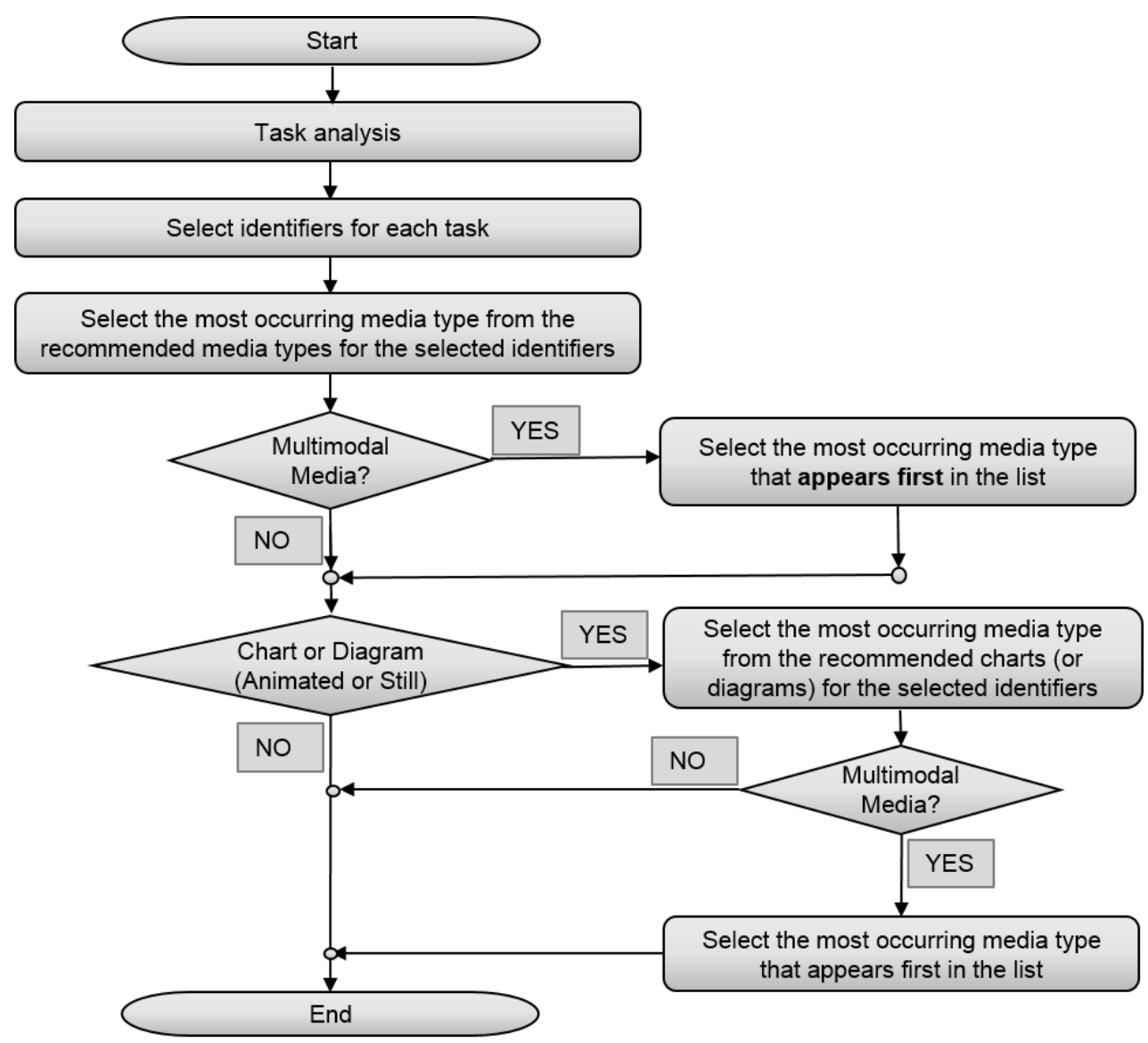

Figure 2: Flowchart outlining the media selection process

types are multimodal (one sentence and one speech output) and sentence is the first occurring media type.

Task 2 (Demonstrate how planetary revolution works): This task requires the demonstration of a process. Looking through the list of identifiers, we have selected the following identifiers:

- Demonstration: This was selected because the task involves showing how planetary revolution works.

- Model: This was selected because presenting this learning concept requires us to rescale the planets for presentation. It will require a model of the real planets.

- Motion: This was selected because the learning concept intends to illustrate the movement (motion) of planets around the sun.

- Object: This was selected because the planets are physical objects.

When we collate the recommended media types for these identifiers, we find that animated diagram is the most occurring media type. This means we need to use an animated diagram to demonstrate the revolution of the planets. Although we have now identified the suitability of the use of an animated diagram for this educational material, we do not know what type of diagram we would need to animate, so we need to go through the second level media selection. Looking through the list of identifiers for diagrams, we have come up with the following: -

- Object: This was selected because the planets are physical objects.

- System: This was selected because a mode of operation is being described.

- Spatial: This was selected because the concept being described has spatial attributes (planets are located in space). 
When we collate the recommended diagram types for these identifiers, we find that schematic diagram is the most occurring diagram type. This means we need to use an animated schematic diagram to demonstrate the revolution of the planets. It is important to note that when the selection process yields an animated chart or an animated diagram from the first level selection, we have to go through the second level selection, determine the media type (chart or diagram) to use and add animation to it.

This simple demonstration shows how we can create educational materials using this framework. We started by defining the tasks for the learning material development and then used the relevant information identifiers to select an appropriate media type to present the learning material.

\section{DISCUSSION, CONCLUSION AND FUTURE WORK}

The positive effects of multimedia on learning have been highlighted in numerous studies (e.g. [1], [2], [3]). Researchers have also highlighted the effects of different media types on learning ([12], [23]) and work has been done to produce guidelines for designing multimedia educational materials (e.g. [14]). In this paper, we have introduced a media taxonomy which presents a way to classify available media types (both existing and new types). We have also presented a framework for the selection of media and demonstrated how it could be used in the design of educational materials, suitable for e-learning systems. Media selection is a very important part of educational material design and it is key to effective learning. It is evident from research that the use of inappropriate media types in educational material design can adversely affect learning [12] so the framework we have presented could help educational material developers select a specific and appropriate media type to present information. While it is not possible to incorporate every existing media type into this framework, the framework contains some generic media types that could be used where a more specific media type is not available. For example, the framework does not contain a media type for chemical structural diagrams normally used in the study of chemical compounds (which can be considered to be a sub type of schematic diagrams), but it will recommend a schematic diagram for learning tasks that involve the presentation of chemical formula. The framework operates in tandem with the media taxonomy and this allows the framework to be easily extended. This extension can be done by introducing more information identifiers and mapping the identifiers to the relevant media types (or incorporating new media types), although it is important to highlight that there is a trade-off between the specificity of the media types recommended by this framework, and its ease of use.

One limitation of the framework is that it requires the users to fully understand how to map the learning concepts to the information identifiers. Though gaining a full understanding of this mapping may take some time, it can get easier with practice. It is also important to note that the scope of this framework is currently limited to the design of educational material for e-learning. The framework may not be suitable for other methods of delivering learning, such as printed books because media types like animations and speech cannot be used on print. Furthermore, some of the media types provided have room for further expansion. For example, the framework suggests a UML diagram for software object presentation but it does not suggest what type of UML diagram to use. This was done to simply the ease of use of the framework. Guides or reference manuals could be used in such situations (e.g. [24]).

In summary, this framework answers questions about "what media to use" in educational multimedia design, however, it does not describe how to use the selected media type. As a result, future work will explore the extension of the framework so as to answer the "how" in relation to the use of selected media and the end goal will be to incorporate the complete framework into a tool that can be used to build effective learning materials for e-learning systems.

\section{REFERENCES}

[1] Najjar, L. (1998). Principles of educational multimedia user interface design. Human Factors: The Journal of the Human Factors and Ergonomics Society, 40(2), pp.311-323..

[2] Mayer, R. (2001). Multimedia learning. New York: Cambridge University Press.

[3] Mayer, R. (2003). The promise of multimedia learning: using the same instructional design methods across different media. Learning and instruction, 13(2), pp.125-139.

[4] Holmquist, Selma, and N. Hari Narayanan (2002). "An integrated architecture for tightly coupled design and evaluation of educational multimedia." Information Sciences 140, no. 1 pp.127-152. 
[5] Teoh, B. and Neo, T. (2006). Innovative teaching: Using multimedia to engage students in interactive learning in higher education. In Information Technology Based Higher Education and Training, ITHET'06. 7th International Conference on (pp. 329-337). IEEE.

[6] Moreno, R. and Mayer, R. (1999). Cognitive principles of multimedia learning. Journal of Educational Psychology, 91, pp.358-368.

[7] Low, R. and Sweller, J. (2005). The modality principle in multimedia learning. The Cambridge handbook of multimedia learning, 147, pp.158.

[8] Clark, J. and Paivio, A. (1991). Dual coding theory and education. Educational psychology review, 3(3), pp.149-210.

[9] Tabbers, H., Martens, R. and Van Merriënboer, J. (2001). The modality effect in multimedia instructions. In Proceedings of the 23rd annual conference of the Cognitive Science Society (pp. 1024-1029).

[10] Harskamp, E., Mayer, R. and Suhre, C. (2007). Does the modality principle for multimedia learning apply to science classrooms? Learning and Instruction, 17(5), pp.465-477.

[11] Clark, Ruth C., and Richard E. Mayer (2011). E-learning and the science of instruction: Proven guidelines for consumers and designers of multimedia learning. John Wiley \& Sons, 2011.

[12] Bhowmick, A., Khasawneh, M., Bowling, S., Gramopadhye, A. and Melloy, B. (2007). Evaluation of alternate multimedia for web-based asynchronous learning. International Journal of Industrial Ergonomics, 37(7), pp.615-629.

[13] Heller, R. S., Martin, C. D., Haneef, N., and Gievska-Krliu, S. (2001). Using a theoretical multimedia taxonomy framework. Journal on Educational Resources in Computing (JERIC), 1(1es), 6.

[14] Sutcliffe, A. G., Kurniawan, S., and Shin, J. E. (2006). A method and advisor tool for multimedia user interface design. International Journal of Human-Computer Studies, 64(4), 375-392.

[15] Daft, R.L. and Lengel, R.H. (1986). Organizational information requirements, media richness and structural design. Management science, 32(5), pp.554-571.

[16] Sun, P.C. and Cheng, H.K. (2007). The design of instructional multimedia in e-Learning: A Media Richness Theory-based approach. Computers \& education, 49(3), pp.662-676.

[17] Bartsch, R.A. and Cobern, K.M. (2003). Effectiveness of PowerPoint presentations in lectures. Computers \& Education, 41(1), pp.77-86.

[18] Sugrue, B. and Clark, R.E. (2000). Media selection for training. Training and retraining: $A$ handbook for business, industry, government, and the military, pp.208-234.

[19] Kozma, R.B. (1991). Learning with media. Review of educational research, 61(2), pp.179-211.

[20] ISO, (2002). ISO 14915-1:2002. Software ergonomics for multimedia user interfaces - Part 1: Design principles and framework. International Standards Organisation.

[21] Johnson, P., Johnson, H., Waddington, R. and Shouls, A. (1988). Task-related knowledge structures: analysis, modelling and application. University of London. Queen Mary College. Department of Computer Science and Statistics.

[22] Annett, J. (2003). Hierarchical task analysis. Handbook of cognitive task design, pp.17-35.

[23] Sahasrabudhe, V., and Kanungo, S. (2014). Appropriate media choice for e-learning effectiveness: Role of learning domain and learning style. Computers \& Education, 76, 237-249.

[24] Rumbaugh, J., Jacobson, I. and Booch, G. (2004). Unified Modeling Language Reference Manual, The. Pearson Higher Education. 